\title{
Potential mechanisms for anisotropy in ice-penetrating radar data
}

\author{
R. DREWS, ${ }^{*}$ O. EISEN, D. STEINHAGE, I. WEIKUSAT, S. KIPFSTUHL, F. WILHELMS \\ Alfred Wegener Institute for Polar and Marine Research (AWI), Bremerhaven, Germany \\ E-mail: reinhard.drews@awi.de
}

\begin{abstract}
Radar data (center frequency $150 \mathrm{MHz}$ ) collected on the Antarctic plateau near the EPICA deep-drilling site in Dronning Maud Land vary systematically in backscattered power, depending on the azimuth antenna orientation. Backscatter extrema are aligned with the principal directions of surface strain rates and change with depth. In the upper $900 \mathrm{~m}$, backscatter is strongest when the antenna polarization is aligned in the direction of maximal compression, while below $900 \mathrm{~m}$ the maxima shift by $90^{\circ}$ pointing towards the lateral flow dilatation. We investigate the backscatter from elongated air bubbles and a vertically varying crystal-orientation fabric (COF) using different scattering models in combination with ice-core data. We hypothesize that short-scale variations in COF are the primary mechanism for the observed anisotropy, and the $900 \mathrm{~m}$ boundary between the two regimes is caused by ice with varying impurity content. Observations of this kind allow the deduction of COF variations with depth and are potentially also suited to map the transition between Holocene and glacial ice.
\end{abstract}

\section{INTRODUCTION}

Efforts are underway to illuminate the ice sheets with a polarimetric airborne system as a precursor to a potential spaceborne mission (Dall, 2010). It is important to understand the polarization dependence of the backscattered signal and to successfully link it to physical properties within the ice. This delivers input for ice-sheet modeling, that enhances both the correct interpretation of paleo-ice-core records and the predictability of the ice sheets. We analyze polarimetric radio-echo sounding (RES) data from the Antarctic plateau, collected near the German summer station Kohnen in east Dronning Maud Land (DML), Antarctica. The data were acquired with an airplane on the ground, measuring the effect of varying polarization as the airplane followed a circular profile. The backscattered power is strongly related to the antenna orientation (i.e. incident polarization), and backscatter patterns change with depth. We use physical properties gained from a nearby deep ice core to discuss mechanisms for anisotropic scattering. Particularly, we focus on ellipsoidal air bubbles and anisotropic crystal-orientation fabric (COF). The ice core was drilled within the European Project for Ice Coring in Antarctica (EPICA) and is referred to as EDML. The drill site is located near a forking ice-divide in a flank-flow setting. An overview of the scientific program at Kohnen station and major results from the EDML ice core are given by Oerter and others (2009).

The polarization of the emitted radar signal is parallel to the airplane heading. In the upper $900 \mathrm{~m}$ the radar backscatter is strongest when the polarization is roughly perpendicular to the nearby ice divide. The orientation of maximum backscatter changes in direction by $90^{\circ}$ at $\sim 900 \mathrm{~m}$ depth, coinciding with the bubble/air-hydrate clathrate transition (under increasing pressure the air bubbles turn into a second solid phase of crystalline air hydrates, also called clathrates) and the transition from Holocene ice to ice from the last glacial period. The polarization dependence exhibits primarily a $180^{\circ}$ symmetry. The aim of this study is to pinpoint a mechanism causing the anisotropy observed in the RES data, and to elucidate the capability of polarimetric

* Present address: Département des Sciences de la Terre et de I'Environnement, Université Libre de Bruxelles, Brussels, Belgium.
RES surveys for the profiling of stress/strain regimes along the vertical.

RES measurements over ice sheets record reflections from dielectric non-uniformities. Density variations are dominant at shallow depths and alter the real and imaginary part of the complex dielectric constant $\left(\varepsilon=\varepsilon^{\prime}-\mathbf{i} \varepsilon^{\prime \prime}\right.$, where $\left.\mathrm{i}^{2}=-1\right)$. Changes in electrical conductivity influence the imaginary part and can be separated from density changes at intermediate depths and below, where density variations are negligible. Both effects often produce laterally coherent internal reflection horizons in the radar data. The dependence of the backscattered signal on the polarization of the incoming wave was noted in the early days of radio-glaciology. Doake (1981) provides an overview. The backscattered signal appeared elliptically polarized, and showed a varying amplitude, depending on the respective azimuth orientation of receiving and transmitting antennas. Using solutions of the Maxwell equations in layered media with a tensorial form for the dielectric properties (Hargreaves, 1977, 1978; Doake, 1981), this could be explained in terms of birefringence and anisotropic reflection coefficients. Hargreaves (1978) proposed as a mechanism for the birefringence the dielectric anisotropy of the single ice crystal in combination with preferred COF. The dielectric anisotropy has been confirmed in subsequent studies (Fujita and others, 1993, 2000; Matsuoka and others, 1997), which found a difference in the real part of the dielectric constant of $\varepsilon_{\|}^{\prime}-\varepsilon_{\perp}^{\prime} \approx 0.035$, for the components parallel $(\|)$ and perpendicular $(\perp)$ to the crystal's $c$-axis. The relative permittivity ranges between 3.1 and 3.2, with small dependences on temperature and frequency.

Numerous multi-frequency and multi-polarization measurements have been performed since then to infer various COF types from RES measurements (Fujita and Mae, 1993; Liu and others, 1994; Fujita and others, 1999, 2003, 2006; Siegert and Kwok, 2000; Doake and others, 2002; Matsuoka and others, 2003, 2004; Eisen and others, 2007; Wang and others, 2008; Dall, 2010; Kravchenko and others, 2011). Variations in density, electrical conductivity and changing COF are now accepted to be the major mechanisms producing RES horizons. Nevertheless, predominantly due to a lack of ice-core data, uncertainties 

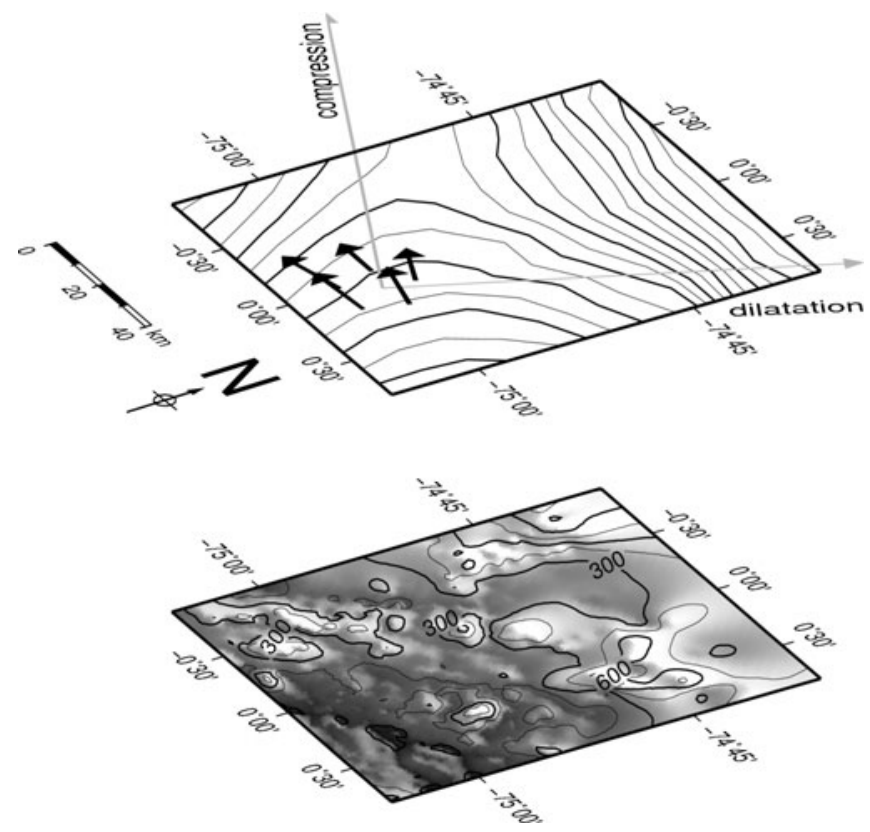

Fig. 1. Map of surface elevation, ice flow (Wesche and others, 2007; black arrows), and bedrock topography (Steinhage and others, 2001). The EDML drill site is at the origin of the light-gray arrows, indicating the principal directions of compression and dilatation.

about the nature of the anisotropic reflection mechanisms remain. In particular, effects from volume scattering have so far been largely neglected. Independent of the specific mechanism for the polarization dependence, the theory of birefringence and anisotropic reflections is well understood.

In birefringent media, waves are refracted depending on the incoming polarization. The solution of the corresponding wave equation results in two characteristic waves, the ordinary wave and the extraordinary wave, which are perpendicularly polarized and generally differ in wave speed. The ordinary wave is refracted according to Snell's law, which does not hold for the extraordinary wave. In uniaxial media (i.e. two out of three principal components of the dielectric tensor are equal) an ordinary wave and an extraordinary wave superimpose. In biaxial media (i.e. all three principal components of the dielectric tensor differ) two extraordinary waves are generated. Typical polarimetric experiments on ice sheets emit an electromagnetic pulse and record amplitude variations of the reflected signal as a function of the azimuth antenna orientation. We discuss only the co-polarized case, which means that the emitting and receiving antennas remain parallel. As described by Hargreaves (1977), the azimuth dependency of the backscattered power is a function of both anisotropic scattering and birefringence, which have different symmetries. In birefringence, the amplitude variation is caused by the superposition of the two characteristic waves with a phase shift. The phase shift depends on the depth-integrated difference in dielectric components perpendicular to the propagation direction. The latter causes a $90^{\circ}$ periodicity, since the magnitude of the difference remains the same for a $90^{\circ}$ rotation of the coordinate system. Anisotropic scattering, however, has a periodicity of $180^{\circ}$, since the corresponding rotation of the antennas at the surface results in an indistinguishable setup. Apart from some special cases (e.g. a fully isotropic COF or a perfect single-maximum COF aligned with the direction of propagation), polar ice is generally birefringent. Anisotropic scattering requires anisotropic gradients in the dielectric properties, for example due to a vertically varying COF, aligned and non-spherical inclusions, or a preferred roughness direction at the interfaces of internal layers. Birefringence and anisotropic scattering are related, and when combined lead to a complex amplitude variation with changing antenna orientation. This can be simulated for the different cases with matrix-based models (Doake, 1981; Fujita and others, 2006; Matsuoka and others, 2009). However, sometimes either a $90^{\circ}$ or a $180^{\circ}$ periodicity in backscattered power is dominant; this allows workers to focus solely either on birefringence or on anisotropic scattering, respectively.

\subsection{Site characteristics and previous studies}

We consider a polarimetric dataset that was acquired along a circle (which changes the antenna's azimuth orientation continuously) with an RES system operating in a co-polarized mode. In the following, we refer to this dataset as the circular RES profile. The location of the measurements is in Dronning Maud Land, $\sim 500 \mathrm{~m}$ downstream of the deep-drilling site for the EDML ice core (Fig. 1). The topography is smooth, with gradients of a few meters per kilometer. The area contains three main ice divides, which merge in a triple junction. The drill site is close to one arm of the forking divides, and the entire setting is in a flank-flow regime. Ice thickness at the drill site is $2774 \mathrm{~m}$ and local surface velocities average $<1 \mathrm{~m} \mathrm{a}^{-1}$ (Wesche and others, 2007).

The bedrock topography was mapped with a dense grid of RES profiles (Steinhage and others, 1999, 2001) during pre-site surveys. After the ice core was drilled, Eisen and others (2006) used data from dielectric profiling and a forward model to link several internal reflectors in the RES data to prominent changes in electrical conductivity. Two of those reflectors (referred to as h1 and h2) are discussed below. Using the same circular RES profile as presented here, a subsequent study (Eisen and others, 2007) showed that another localized reflector at $2035 \mathrm{~m}$ depth is caused by a drastic change in COF which is observed in ice-core data. Linescan images from the ice core show the stratigraphy on a sub-centimeter resolution. The stratigraphy appears increasingly disturbed with greater depths. This suggests that the disappearance of internal reflection horizons below $\sim 2100 \mathrm{~m}$ depth may be caused by stratigraphic disturbances on a larger scale (Waddington and others, 2001; Drews and others, 2009).

Wesche and others (2007) derived an elevation model as well as surface velocities with corresponding stress/strain rates, based on a pentagon-shaped stake network. The flow regime is characterized by along-flow compression and lateral dilatation. The maximal principal strain-rate component has a magnitude of $-1.85 \times 10^{-4} \mathrm{a}^{-1}$ and points towards $24^{\circ} \mathrm{N}$. The minimal principal component points towards $114^{\circ} \mathrm{N}$ with a magnitude of $2.32 \times 10^{-4} \mathrm{a}^{-1}$. Ueltzhöffer and others (2010) investigated size, number and shape of the air bubbles in vertical cuts from the ice core. The transition of air bubbles into clathrates starts at $\sim 700 \mathrm{~m}$ and progresses rapidly below $800 \mathrm{~m}$. Typical effective bubble radii cluster around $0.1 \mathrm{~mm}$, decreasing with depth, similar to the volume fraction, which ranges from $\sim 0.004(200 \mathrm{~m}$ depth) to $\sim 0.001$ (900 $\mathrm{m}$ depth). In vertical cuts, it was found that the bubbles are elongated, with eccentricities varying from 0.3 to 0.8 . Due to the lack of continuous 
azimuth control of the ice core, no correlation with ice flow is possible. Bendel (2009) did some further analysis on vertical cuts and found that number density and aspect ratio vary on centimeter scales, in particular for glacial ice, but also at shallower depths.

Crystal-orientation fabric studies on horizontal and vertical thin sections exploit the birefringent nature of the single crystals by using crossed polarizers to determine the $c$-axis orientation. The orientation distribution is often visualized using Schmidt diagrams, which project the piercing point of the $c$-axes through a hemisphere onto a plane. By fitting an ellipsoid that has the same moment of inertia as the distribution of piercing points (with imaginary masses attached), the COF types can be characterized with three eigenvalues, $\lambda_{1}, \lambda_{2}, \lambda_{3}$, and their corresponding eigenvectors. The eigenvalues define the ellipsoid's shape, the eigenvectors its orientation. As explained below, it can be assumed that the first two eigenvectors are in the horizontal plane, with the third eigenvector pointing vertically. Two specific COF types are the single-maximum and the verticalgirdle distribution. In a perfect single-maximum distribution, all $c$-axes point vertically $\left(\lambda_{1}=\lambda_{2}=0, \lambda_{3}=1\right)$. In a vertical-girdle $\operatorname{COF}\left(\lambda_{1}<\lambda_{2}<\lambda_{3}\right)$, the $c$-axes cluster in a deformed cone around the vertical axis. More details of the fabric measurements and the results are presented below.

\section{DATA ACQUISITION AND OBSERVATION}

The radar data were acquired in 2003 with an RES system designed for the airborne sounding of ice sheets (Nixdorf and others, 1999). The short backfire antennas emit bursts with a pulse length of 60 or $600 \mathrm{~ns}$ at a center frequency of $150 \mathrm{MHz}$. The electric field vector oscillates parallel to the airplane's heading. For this study, the short pulse was used to increase the vertical resolution, at the cost of total penetration depth. The antennas are fixed on the wings. To investigate the response with varying incoming polarization, the airplane (a Dornier 228) taxied on the ground in a circle. In this co-polarized mode, the emitted and recorded polarization planes were always parallel and rotated continuously around the vertical while the airplane moved along the circle. Traveltime-to-depth conversion was done with the interpolation of tie points from ice-core dielectric profiling data with RES horizons (Eisen and others, 2006). The angle heading is defined as the heading angle of the aircraft in respect to true north. It depicts the angle of the electric field vector.

The backscattered power along the circular profile is displayed for the depth interval of 200-1400 m in Figure 2a. The diameter of the circle is $\sim 100 \mathrm{~m}$ and the profile consists of 170 traces with an average trace spacing of $1.8 \mathrm{~m}$. Each trace is a tenfold stack of shots and no further processing has been applied. The dominant feature is the overlying sinusoidal variation with changing azimuth of the two antennas. The signal has a clear $180^{\circ}$ periodicity and splits in two parts along the vertical: from $\sim 200$ to $900 \mathrm{~m}$ depth, the maxima develop at a heading angle of $\sim 206^{\circ} \mathrm{N} / 26^{\circ} \mathrm{N}$ (note that the heading is tangential to the circle). Below $900 \mathrm{~m}$, the maxima shift by $90^{\circ}$, and occur at a heading of $\sim 116^{\circ} \mathrm{N} / 296^{\circ} \mathrm{N}$. We define the two depth intervals as anisotropic reflections zones (ARZ) 1 and 2, respectively. The different directions are shown in Figure $2 b$. The strain ellipsoid marks the directions of compression and dilatation given by Wesche and others (2007). The directions

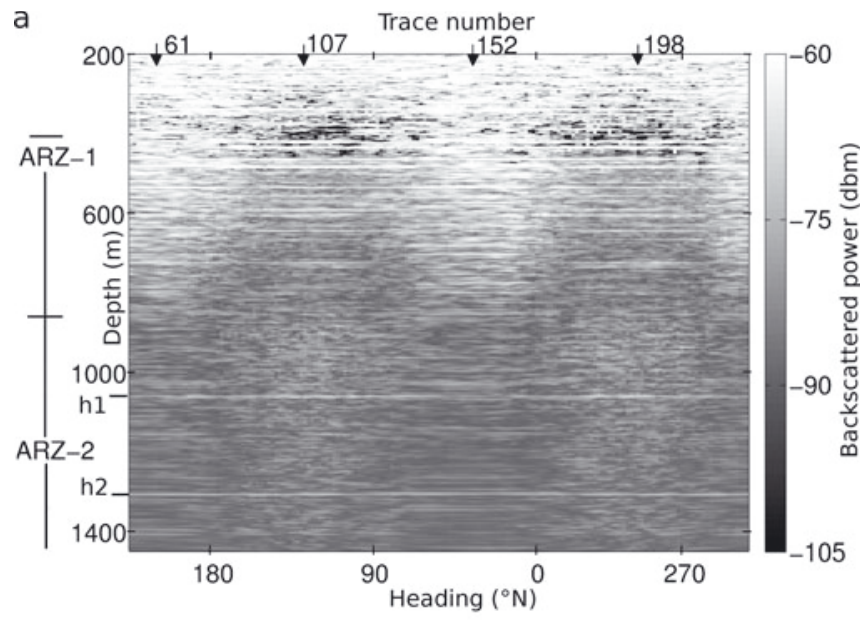

b

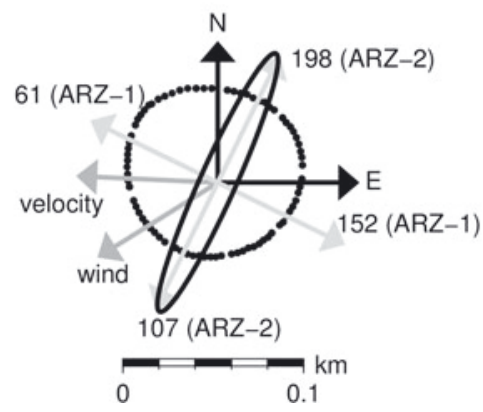

Fig. 2. (a) The backscattered power of the circular profile varies sinusoidally. The two horizontal lines marked h1 and h2 are internal reflection horizons linked to prominent peaks in dielectric profiling data by Eisen and others (2006). (b) Top view of (a) marking the shot point coordinates of the circular RES profile (black dots). The dark-gray arrows depict the main flow direction of ice together with main wind direction; strong wind events turn to more northerly directions. The light-gray arrows mark the extrema in backscatter on the circle for the different zones (numbers are trace numbers of (a)). The electric field vector is tangential to the circle. The strain ellipsoid (dark ellipse) indicates the directions for the principal components of compression and dilatation, following Wesche and others (2007). These directions correspond well with the backscatter extrema observed in the RES profile.

of minimal and maximal backscatter correspond well with the principal axes of the strain ellipsoid.

\section{MECHANISMS FOR POLARIZATION- DEPENDENT BACKSCATTER}

As described above, the polarization dependence of the backscattered radar signal can principally be induced by birefringence and by anisotropic scattering. Birefringence is a direct consequence of the ice crystal's dielectric anisotropy. Anisotropic scattering requires anisotropic changes in the dielectric properties. The two effects can be distinguished on the basis of symmetry.

To better visualize the symmetry, vertically averaged intervals of $200 \mathrm{~m}$ thickness are shown in Figure 3. Due to absorption and spherical spreading, the averages within the individual intervals are biased towards shallower values, but this does not affect the symmetry. The $180^{\circ}$ periodicity in backscattered power appears overlaid by a $90^{\circ}$ pattern resulting in two small local maxima between the main maxima mentioned above. From these two symmetries we 


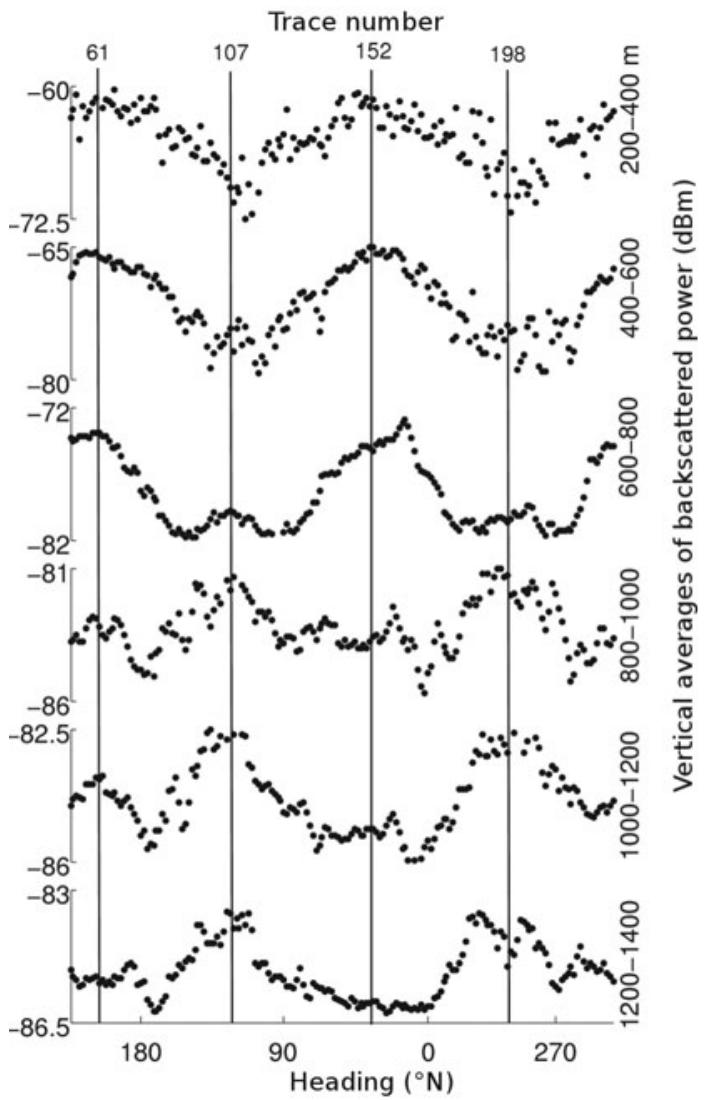

Fig. 3. Vertical averages of backscattered power $(\mathrm{dB} \mathrm{m}$, i.e. referenced to $1 \mathrm{~mW}$ ) in $200 \mathrm{~m}$ intervals from 200 to $1400 \mathrm{~m}$ depth plotted against heading (bottom axis) and trace number (top axis). The main maxima with the $180^{\circ}$ periodicity are partly interweaved with small maxima with a $90^{\circ}$ offset.

deduce that both birefringence and anisotropic scattering influence the azimuth dependency of the backscattered power. However, anisotropic scattering is dominant and causes the larger amplitudes of the $180^{\circ}$ cycle. The interweaved smaller maxima have a $90^{\circ}$ offset, so both effects appear to have similar principal axes, that do not change noticeably with increasing depth. Due to the dominance of the $180^{\circ}$ periodicity, we focus on potential anisotropic scattering mechanisms.

The appearance of two anisotropic reflection zones has also been observed in other studies. Fujita and Mae (1993) compared data from a $179 \mathrm{MHz}$ ground-based radar with fabric measurements from the ice core at Mizuho station, Antarctica. They observed high amplitudes in the upper part of the ice column with the antennas parallel to the flowline, and lower amplitudes for the perpendicular arrangement. Below $800 \mathrm{~m}$ depth, a similar pattern appears, but in the reversed order. The Mizuho ice core does not reach the bedrock and ends at $\sim 700 \mathrm{~m}$. This depth is above both the bubble/clathrate transition (Shimada and Hondoh, 2004) and the transition from the Holocene into the last glacial period (Nakawo and others, 1989) at this site. Using different frequencies, pulse lengths and antenna orientations in the same study area, Fujita and others (2003) confirmed the previous findings of changing anisotropy with depth. They proposed as mechanisms for the upper anisotropy, a varying cluster strength for the girdle-type fabric, and for the lower anisotropy layered strata composed of girdle and single-pole transitions. Using similar techniques Matsuoka and others (2003) also observed the two different zones along a ground-based traverse from Dome Fuji, Antarctica, towards the Shirase Glacier drainage basin. For the lower anisotropy they supported the idea of transitions from girdle to single-pole COF as the primary mechanism. An airborne survey (Matsuoka and others, 2004) identified high scattering in the deeper layers of convergent and compressional iceflow regimes when the polarization was perpendicular to the flow direction. Using a matrix model, Fujita and others (2006) discriminated between birefringence and anisotropic scattering at Mizuho and Dome Fuji. In both cases aligned COF and changes thereof were assumed to be the main mechanism for the observed polarization dependence. Based on the previous studies, the scenario for the two anisotropic reflection zones near Mizuho station is:

One of the dielectric tensor's principal directions is close to vertical. A varying cluster strength of a girdle-type COF translates into a variation of the first eigenvalue in the horizontal plane. This increases the backscatter for an incoming polarization parallel to the corresponding principal axes. There are no significant changes in the second eigenvalue in the horizontal plane.

At larger depths, girdle to single-pole transitions change the vertical, and the second horizontal principal component. The latter increases backscatter perpendicular to the direction observed in the upper interval.

If only one eigenvalue varies in the horizontal, a rotation of the principal axis with increasing depth may result in a similar pattern. The problem with the hypotheses is that only a single study (Eisen and others, 2007) directly linked (an exceptional large) short-scale variation in COF from icecore data to a reflector in RES data in the lower part of the ice sheet. For the middle to upper depths, vertically higher resolved COF data in conjunction with polarimetric RES measurements are needed to confirm this idea. Therefore, before discussing the effect of variable COF at the EDML drill site, we also consider other possibilities, such as anisotropic inclusions or layer boundaries with a directional roughness.

It can be speculated that patterns that arise on the surface from preferred wind directions are advected to larger depths while preserving their directionality, thus causing anisotropic interfaces between different layers. Birnbaum and others (2010) investigated the formation of snow dunes in the EDML area. They found that strong wind events create aligned wind-shaped features, which can also be seen in the physical properties of shallow firn cores (down to $5 \mathrm{~m}$ depth). The main (katabatic) wind direction is depicted in Figure $2 \mathrm{~b}$, but strong wind events have a more northerly direction (Reijmer and Van den Broeke, 2003) and may align with the backscatter extrema in the radar data. However, deeper ice seems to have no memory of structures caused by deposition at the surface. First analyses of the density and microstructure of firn (Hörhold and others, 2012) indicate that impurities significantly influence, or maybe even control, density and to a large extent the pore-space structure in deeper firn. Characteristic surface features, such as sastrugi or snow dunes seem not to be imprinted in deep ice; in deeper firn at densities between $\sim 630$ and $750 \mathrm{~kg} \mathrm{~m}^{-3}$, density and porosity are rather correlated with the concentration of impurities. It seems reasonable that a preferred roughness will decrease with increasing depths, but the sinusoidal pattern observed in Figure 2 starts at $\sim 200-300 \mathrm{~m}$ and 
sharpens its directional dependence with increasing depth. However, at shallower depths, the quality of the radar data is insufficient and wind-induced anisotropy may also be present in the upper 0-300 m. Polarimetric data from a radar system designed to illuminate the shallow layering of ice, and an improved understanding of densification processes are needed to further investigate this topic.

The effect of enclosed (and possibly distorted) air bubbles on the radar backscatter has been discussed in several studies. Using a spherical shape, a frequency of $35 \mathrm{MHz}$, the dipole approximation and a volume-type radar equation, Robin and others (1969) suggested that air bubbles may have a small but noticeable effect on the backscattered power. Discussing birefringence, Hargreaves (1978) estimated the effect from elongated air bubbles to be too small to explain experimental results. Ackley and Keliher (1979) used data from the Cape Folger ice core, near the coast of East Antarctica, and estimated the effect from the elongated air bubbles to be larger than that from COF variations. They observed layered strata where the bubble elongation corresponded to density fluctuations, suggesting a link to deformation. Alley and Fitzpatrick (1999) analytically described the bubble elongation as an interplay between strain-induced deformation and diffusive restoration. Very elongated bubbles with the long axis being several times the short axis are seen most frequently in ice cores near the coast within high-shear regimes. The observed eccentricities in the EDML ice core are much smaller. Fujita and others (2000) used new COF data and placed the effect from air bubbles on the backscatter lower than the dominating density, electrical conductivity and COF mechanisms.

Three indications motivate us to investigate the effect of air bubbles on the backscatter more closely: (1) Ueltzhöffer and others (2010) showed in vertical cuts that air bubbles at the EDML drill site are vertically deformed. This means that bubbles can maintain an ellipsoidal shape within the ice matrix, and there is reason to believe that they might also be deformed in the horizontal plane; (2) the coincidence of the clathrate transition zone with the transition between the anisotropic reflection zones (ARZ-1 and ARZ-2) and (3) the strain ellipsoid in Figure $2 \mathrm{~b}$ indicates the orientation of deformed bubbles (if surface strain is considered as the exclusive mechanism for bubble deformation). The direction of the long bubble-axis coincides with the direction of maximum backscatter. In Section 3.1 we apply an analytical volume-scattering model to estimate the effect of discrete ellipsoidal scatterers in the ice matrix. In Section 3.2, COF data from the EDML ice core and the corresponding effect on scattering are discussed, in order to differentiate between the different mechanisms.

\subsection{Volume-scattering model for a half-space with discrete ellipsoids}

3.1.1. Model set-up

The analysis presented here is based on Tsang and others (1981). Volume-scattering models of this kind have been developed primarily for satellite applications, for example to classify the backscatter from canopy. We adapt the model for our purpose by changing the boundary conditions and focusing on a nadir-looking case. The geometry is shown in Figure 4. It includes two half-spaces 0 and 1 , with permittivity $\varepsilon_{0}$ for air and $\varepsilon_{1}$ for ice, separated at the boundary $z=0$. Ellipsoids with permittivity $\varepsilon_{\mathrm{s}}$ are randomly positioned in the lower half-space. The approach is based on the radiative

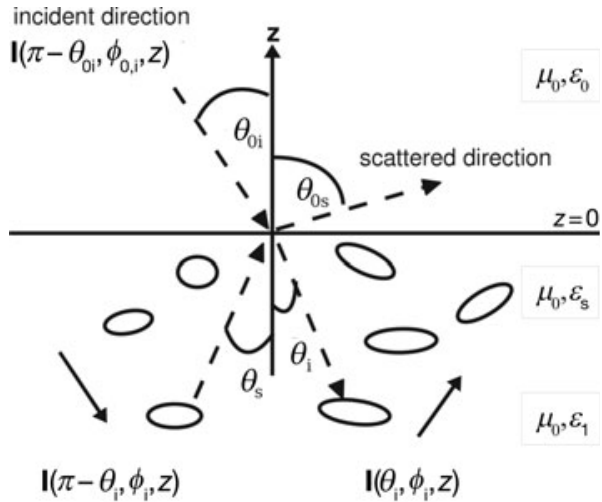

Fig. 4. Sketch for the model of discrete ellipsoidal scatterer, modified after figure 1 of Tsang and others (1981).

transfer theory, which deals with specific intensities, I, rather than the electromagnetic fields, $\mathbf{E}$ (see, e.g., Tsang and others, 2000, for an introduction). The specific intensities are $4 \times 1$ vectors. The first two components are the vertically (index v) and the horizontally (index h) polarized intensities $I_{v}$ and $l_{\mathrm{h}}$. Although for nadir incidence the distinction of vertical and horizontal becomes ambiguous (Doake and others, 2002), we keep the notation to remain consistent with other models. The indices $\mathrm{v}$ and $\mathrm{h}$ thus represent polarization types corresponding to orthogonal orientations of the (COpolarized) antennas in the horizontal plane. The third and fourth components depict the correlation between vertical and horizontal polarization and enable the description of any polarization state. Using the modified Stokes vectors, the specific intensities are given by

$$
\mathbf{I}=\left[\mathrm{I}_{\mathrm{h}}, \mathrm{I}_{\mathrm{v}}, 2 \operatorname{Re}\left(E_{\mathrm{v}} E_{\mathrm{h}}^{*}\right), 2 \operatorname{Im}\left(E_{\mathrm{v}} E_{\mathrm{h}}^{*}\right)\right]
$$

where $\mathbf{I}=[1,0,0,0]$ corresponds to the fully vertically polarized and $\mathbf{I}=[0,1,0,0]$ to the fully horizontally polarized case; ${ }^{*}$ depicts the complex conjugate and Re and Im refer to the real and imaginary part, respectively. The changes of the incoming intensity with depth are caused by extinction and scattering. The extinction is described with the extinction matrix, $\underline{\mathbf{k}}_{\mathbf{e}}=\underline{\mathbf{k}}_{\mathbf{a}}+\underline{\mathbf{k}}_{\mathbf{s}}$, as a sum of absorption (index a) and scattering (index s) away from the receiver. We consider absorption to be isotropic. The phase matrix, $\underline{\mathbf{P}}$, operates for scattering from direction $\theta^{\prime}, \phi^{\prime}$ into the direction $\theta, \phi$. All matrices are $4 \times 4$. Shape, dielectric contrast and spatial orientation of the ellipsoids define the individual components of $\underline{\mathbf{P}}$. They can be calculated in a Rayleigh approximation using scattering dyads (Tsang and others, 1981). The Euler angles, $\alpha, \beta, \gamma$, define the orientation of the randomly positioned bubbles. The rotation around the $z$-axis corresponds to the angle $\alpha$. For a definition of all angles see figure 2 of Tsang and others (1981). The principal axes of the ellipsoids are defined as $a, b$ and $c$; $a$ and $b$ are in the horizontal plane when $\alpha=\beta=\gamma=0$. Each element is averaged over a common orientation density distribution, $\psi(\alpha, \beta, \gamma, \Delta \alpha, \Delta \beta, \Delta \gamma)$, which quantifies the degree of alignment for the bubbles. For simplicity, $\psi$ is assumed to be box-like in all three angles, i.e. within the corresponding $\Delta$-values all angles are equally probable, while outside the $\Delta$-values the probability is zero. The fully aligned and the fully random cases for $\alpha$ correspond to $\Delta \alpha=0^{\circ}$ and $\Delta \alpha= \pm 90^{\circ}$, respectively. For non-spherical shapes, $\underline{\mathbf{P}}$ is non-diagonal and the different components in 

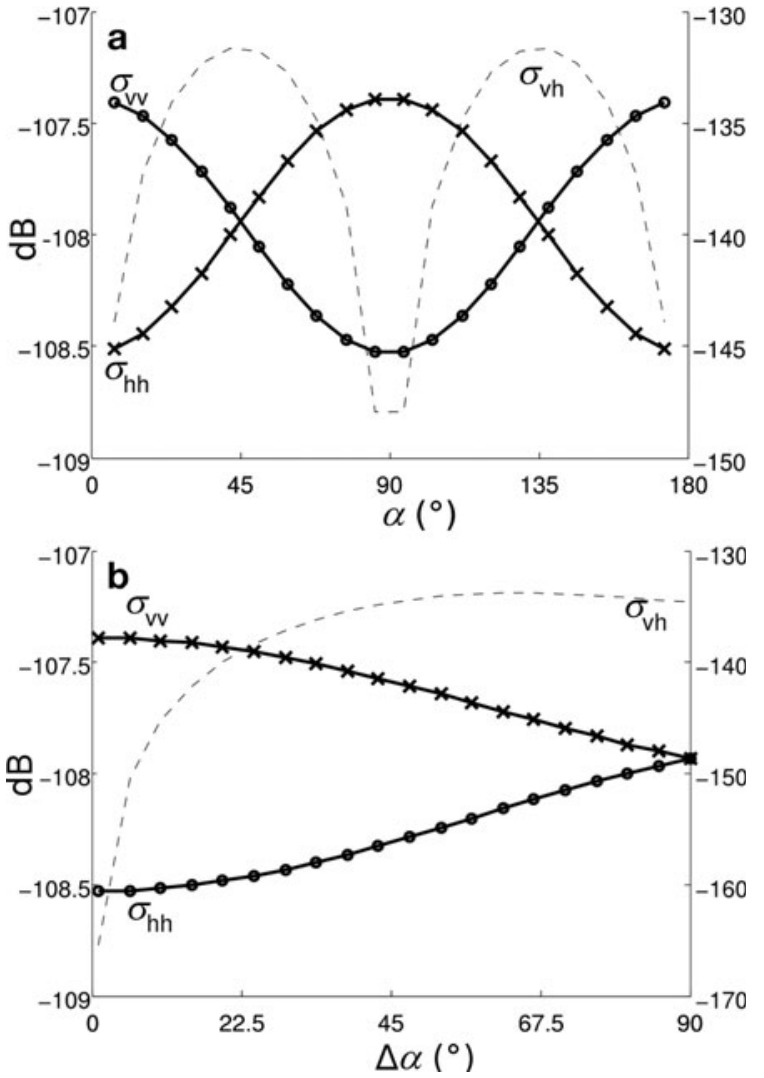

Fig. 5. Model outcomes for the backscatter coefficients (hh, vv with solid curves and vertical axis on the left, hv with dashed curve and vertical axis on the right). (a) $\alpha$ rotates in the horizontal plane while $\beta$ and $\gamma$ remain fixed. No orientation density distribution is applied. This corresponds to the fully aligned case $(\Delta \alpha=\Delta \beta=\Delta \gamma=0)$. (b) Increasing disorder is simulated for $\alpha=\beta=\gamma=0$ and increasing $\Delta \alpha$. Parameters for (a) and (b) are $\varepsilon_{1}=(3.2+0.0018 \mathrm{i}) \varepsilon_{0}$, $\varepsilon_{\mathrm{s}}=\varepsilon_{0}, \Theta_{\mathrm{oi}}=\phi_{\mathrm{oi}}=0, \mathrm{vol}=0.0001$ (volume fraction), $a=0.15 \mathrm{~mm}, b=c=0.1 \mathrm{~mm}$.

the specific intensity, I, mix, leading to depolarization. The governing equation is

$$
\cos (\theta) \frac{\mathrm{d} \mathbf{I}}{\mathrm{d} z}=-\underline{\mathbf{k}}_{\mathrm{e}} \mathbf{I}(\theta, \phi, z)+\int \mathrm{d} \Omega^{\prime} \underline{\mathbf{P}}\left(\theta, \phi, \theta^{\prime}, \phi^{\prime}\right) \mathbf{I}(\theta, \phi, z) .
$$

The integration is over the solid angle $\mathrm{d} \Omega^{\prime}=\sin \left(\theta^{\prime}\right) \mathrm{d} \theta^{\prime} \mathrm{d} \phi^{\prime}$. The angles are displayed in Figure 4 , with $\phi$ being the azimuthal angle in the horizontal plane.

We first treat the general case of oblique incidence, and then consider the backscattering for a nadir-looking sensor as a special case. The integro-differential equation (1) can be solved using perturbation theory. The different terms of the perturbation series correspond to the different instances of multiple scattering. For backscattering, the incoming direction (index i) equals the scattered direction (index s). In terms of the normalized wavevectors this means $\hat{\mathbf{k}}_{\mathrm{i}}=-\hat{\mathbf{k}}_{\mathrm{s}}$, with $k=2 \pi f \sqrt{\mu \varepsilon}$ (with $\mu$ being the magnetic permeability), where $f$ is the frequency. The backscattering coefficient, $\sigma_{\gamma, \delta}$, quantifies the ratio of incident specific intensity (direction $\hat{\mathbf{k}}_{\mathbf{i}}$ with polarization $\delta=\mathrm{v}$ or h) to backscattered specific intensity (direction $-\hat{\mathbf{k}}_{\mathrm{i}}$ with polarization $\gamma=\mathrm{v}$ or $\mathrm{h}$ ):

$$
\sigma_{\gamma \delta}\left(\hat{k}_{\mathrm{i}},-\hat{k}_{\mathrm{i}}\right)=4 \pi \cos \left(\theta_{\mathrm{oi}}\right) \cdot \frac{I_{\gamma \mathrm{s}}}{I_{\delta \mathrm{i}}}
$$

(see Fig. 2 for the definition of angles). The solution is outlined in the Appendix. The first-order approximation of Eqn (1), marked with superscript (1), corresponds to single scattering. For the nadir-looking geometry $\left(\theta_{\mathrm{oi}}=\theta_{\mathrm{i}}=0 ; \pi+\phi_{\mathrm{i}}=\right.$ $\phi_{\mathrm{oi}}=0$ ) we obtain

$$
\begin{aligned}
\sigma_{\mathrm{vv}}^{(1)} & =2 \pi t_{\mathrm{v} 10}^{2} \frac{\varepsilon_{0}}{\varepsilon_{1}} \frac{P_{11}(0, \pi, \pi, 0)}{k_{\mathrm{v}}}, \\
\sigma_{\mathrm{hh}}^{(1)} & =2 \pi t_{\mathrm{h} 10}^{2} \frac{\varepsilon_{0}}{\varepsilon_{1}} \frac{P_{22}(0, \pi, \pi, 0)}{k_{\mathrm{h}}}, \\
\sigma_{\mathrm{hv}}^{(1)} & =2 \pi t_{\mathrm{v} 10} t_{\mathrm{h} 01} \frac{\varepsilon_{0}}{\varepsilon_{1}} \frac{P_{12}(0, \pi, \pi, 0)}{k_{\mathrm{h}}}, \\
\sigma_{\mathrm{vh}}^{(1)} & =2 \pi t_{\mathrm{h} 10} t_{\mathrm{v} 01} \frac{\varepsilon_{0}}{\varepsilon_{1}} \frac{P_{21}(0, \pi, \pi, 0)}{k_{\mathrm{v}}} .
\end{aligned}
$$

The scattering coefficient is a function of several parameters (e.g. frequency, volume fraction, incidence angles, eccentricities, alignment of bubbles) through the phase matrix. The transmission between air (index 0) and ice (index 1) for the different polarizations is labeled with the power transmission coefficients, $t_{\mathrm{v} i j}$ and $t_{\mathrm{h} i j}$, where $i$ and $j$ can take the values 0 or 1 . Since the air/ice interface is assumed to be isotropic, they play a minor role. The variables $k_{\mathrm{v}}$ and $k_{\mathrm{h}}$ are the first two diagonal elements of the extinction matrix, $\mathbf{k}_{\mathbf{e}}$. Extinction includes off-angle scattering and absorption in ice. The latter is considered with the complex part of the permittivity: $\varepsilon_{1}^{\prime \prime}=\sigma /\left(2 \pi f \varepsilon_{0}\right)$.

We also calculated a second-order solution (Drews, 2009) which treats multiple scattering. However, apart from depolarization effects, this is of minor importance for the parameters used here. The analytical solution for higher-order terms is only feasible for spherical inclusions (or spheroids), and a fully numerical approach or other approximations are needed if higher-order terms are desired.

\subsubsection{Model outcomes}

The polarization dependence of the backscatter coefficient is simulated by keeping the antenna orientation fixed, and rotating the bubbles in the horizontal by changing the values for the Euler angle, $\alpha$. The sinusoidal variation of the backscattering coefficients is shown in Figure $5 \mathrm{a}$ for the case of fully aligned ellipsoids $(\Delta \alpha=\Delta \beta=$ $\Delta \gamma=0$ ). The absolute value of the phase matrix elements is mainly dependent on the ratio of bubble volume to wavelength. The magnitude of the backscatter coefficient is also influenced by absorption. For the examples presented here, we assume an average conductivity of $15 \mu \mathrm{Sm}^{-1}$, as determined by dielectric profiling measurements (Wilhelms and others, 1998; Wilhelms, 2000) from the EDML ice core. The measurements are scaled to the center frequency of the RES system (Eisen and others, 2006). The difference of vertical and horizontal backscatter coefficients depends on the bubble eccentricity and the degree of alignment determined by the orientation density function.

The effect of order and disorder in bubble alignment is shown in Figure 5b. The Euler angles $\alpha=\beta=\gamma=0$ are kept constant with $\Delta \beta=\Delta \gamma=0$. The disorder in the horizontal plane increases with increasing $\Delta \alpha$. For the fully random case $\left(\Delta \alpha=90^{\circ}\right)$ the polarization dependence ceases.

\subsection{Changing crystal orientation fabric}

Crystal orientation fabric data of the EDML ice core were measured in 2005 in a $-20^{\circ} \mathrm{C}$ cold room using an automatic fabric analyzer system (Wilson and others, 2003). The EDML 

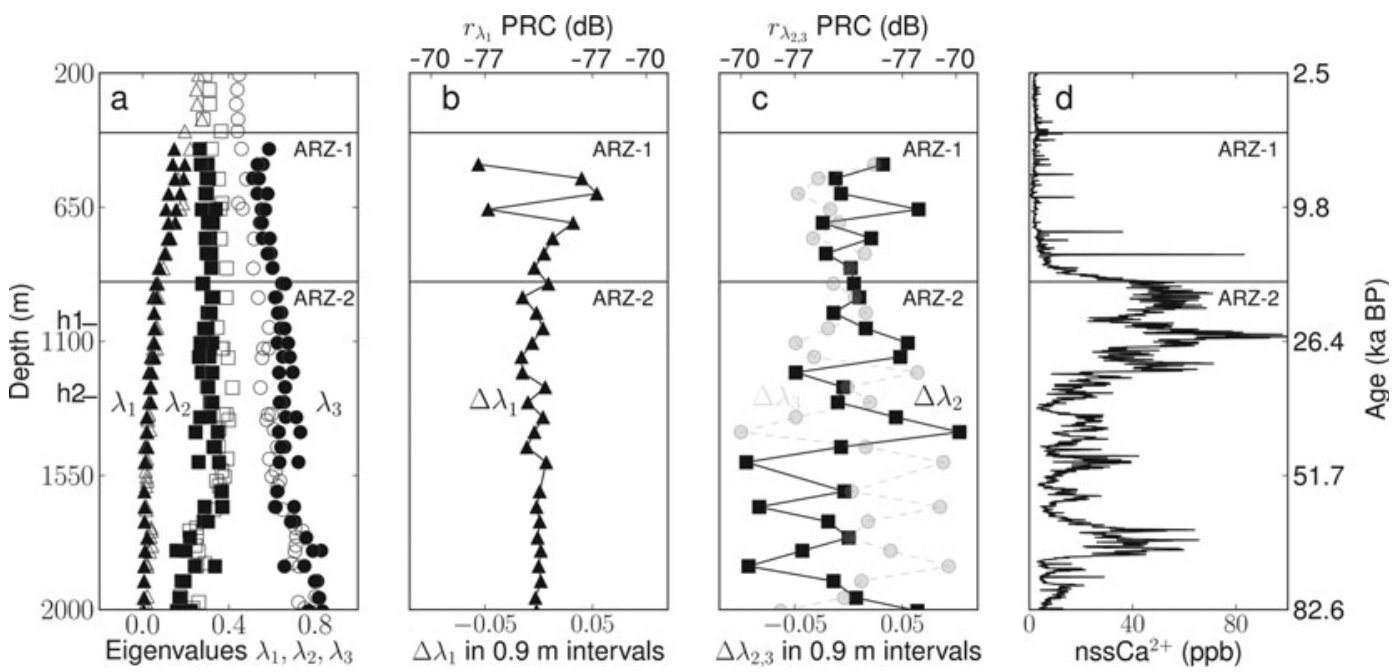

Fig. 6. (a) Crystal orientation fabric data from the EDML ice core in terms of eigenvalues $\left(\lambda_{1}=\right.$ triangle, $\lambda_{2}=$ square, $\lambda_{3}=$ circle; filled symbols for horizontal cuts, open symbols for vertical cuts). The depths of two prominent internal reflection horizons in the radar data are marked h1 and h2. The difference, $\Delta \lambda_{i}$, for horizontal cuts measured in $0.9 \mathrm{~m}$ intervals is displayed in (b) and (c). The top axes mark the corresponding power reflection coefficients (PRCs), as calculated from a two-layer approximation given in Eqn (3). Changes parallel to the propagation direction (i.e. changes in $\lambda_{3}$ ) do not influence the backscatter. (d) Non-sea-salt (nss) $\mathrm{Ca}^{2+}$ concentration as published by Fischer and others (2007) serves as a proxy for impurities within the ice which decrease in concentration during the transition from the last glacial into the Holocene (700-900 m depth).

core was drilled between 2001 and 2004 and was stored at $-30^{\circ} \mathrm{C}$ after transportation to the storage facilities. Thin sections were prepared using a microtome from samples cut horizontally $\left(\sim 0.5 \times 50 \times 50 \mathrm{~mm}^{3}\right)$ and vertically $(\sim 0.5 \times$ $50 \times 100 \mathrm{~mm}^{3}$ ). The automatic fabric analyzer measures the crystal orientation of these samples within $30 \mathrm{~min}$. This is possible due to the full automation of a polarization microscope (Wilen and others, 2003) with a CCD (chargecoupled device) camera acquiring images of the thin section between rotating crossed polarizers from nine different viewing angles. The derived eigenvalues characterizing the COF distribution should, in theory, be independent of the cutting direction. However, in particular for eigenvalues $\lambda_{2}$ and $\lambda_{3}$, an offset between the horizontal and vertical cuts is evident (Fig. 6a, difference between filled and unfilled symbols). We believe this artifact is introduced by the automatic exclusion of grains with a $c$-axis orientation that is difficult to measure (e.g. the $c$-axes being parallel to the section plane). Since the COF is non-random but aligned, these cases occur in different frequencies when the cutting direction is changed. The estimated uncertainty for the magnitude of the individual eigenvalues is 0.1 , but confidence in relative changes of eigenvalues determined with the same method can be considered higher.

The COF in EDML shows a gradual transition from isotropically oriented $c$-axes at shallower depths (down to $\sim 450 \mathrm{~m}$ ) to a broad girdle fabric ( 450-1700 m depth). The eigenvalues in Figure 6 a reflect this transition by evolving from three similar values into three distinct values $\left(\lambda_{1}<\right.$ $\left.\lambda_{2}<\lambda_{3}\right)$. We attribute this to the evolving influence of uniaxial extension and lateral flow dilatation. The girdle fabric narrows from $1700 \mathrm{~m}$ down to $\sim 2000 \mathrm{~m}$ depth, indicated by converging $\lambda_{2}$ and $\lambda_{1}$. This is possibly caused by a transition from dominating horizontal uniaxial extension to bed-parallel simple shear.

Fabric data are measured at intervals of $50 \mathrm{~m}$ or less using vertical cuts. Starting at $450 \mathrm{~m}$ depth, the measurements are complemented with two horizontal cuts within $0.9 \mathrm{~m}$ distance every $50 \mathrm{~m}$. We use this dataset as a proxy for shortscale variations in COF. The difference, $\Delta \lambda_{i}(i=1,2,3)$, within the $0.9 \mathrm{~m}$ interval for the three eigenvalues is plotted for increasing depth in Figure $6 b$ and $c$. In the range of the first anisotropic reflection zone (ARZ-1), the variation of the smallest eigenvalue, $\lambda_{1}$, is noticeable, and decreases towards larger depths. This corresponds to the previously discussed variation in girdle strength for ARZ-1. The opposite behavior becomes apparent for the two other eigenvalues, $\lambda_{2}$ and $\lambda_{3}$, which eventually swing in antiphase parallel to ARZ2 's appearance in the radar data. This implies the tendency of girdle to single-pole transitions in ARZ-2. The relative changes of the eigenvalues considered here are close to the detection limit of the fabric measurements. However, at least at larger depths, the general tendency appears systematic and seems to fit with the effects seen in the radar data, as discussed in Section 3.3. We therefore carry on, but bear in mind that more accurate COF data, preferably at higher vertical resolution, are needed to solidify the later conclusions drawn from this dataset with respect to the interaction with radar waves.

We use a layer approximation to estimate the effect on the backscatter. A premise is that the changes in COF as observed in the ice core are laterally coherent on a scale that is much larger than the illuminated area of a single RES pulse. For larger depths, this has been shown by Eisen and others (2007), who linked a single RES reflector to changes in COF and traced it laterally for a few kilometers. For the intermediate depths this cannot easily be done, because changes in COF are less distinct. However, the evolution of $\mathrm{COF}$ is related to strain rates, and there are also indications that a varying impurity content influences the crystal size and thus the formation of COF (Thorsteinsson, 1996). Both effects favor a laterally coherent stratification of COF. Prominent internal reflection horizons ( $\mathrm{h} 1$ and $\mathrm{h} 2$ ) are visible at 1068 and $1312 \mathrm{~m}$ depth in Figure 2a. Eisen and others (2006) linked both horizons to (isotropic) reflectors caused by changes in electrical conductivity and we would expect - if 
at all - brightness variation in the polarimetric data with the symmetry of birefringence. The internal horizon h2 appears to be independent of the incoming polarization, whereas h1 shows some brightness variations, with minima and maxima coinciding with the characteristics of ARZ-2. Insufficient data are available to fully explain this. It is possible that impurity content in ice impacts the COF type. Changes in electrical conductivity may be accompanied by laterally coherent changes in COF, leading to a polarization dependence of the corresponding reflection horizon. It seems that the layer approximation is certainly simplified but not unrealistic.

In order to characterize the dielectric properties of the individual layers, we need to know the principal directions associated with the eigenvalues. This is difficult, since no continuous azimuth control in the horizontal plane is available for the ice core. However, the third eigenvector seems to be in the vertical, which becomes apparent in some Schmidt diagrams by an accumulation of points in the center of the girdle. We therefore tentatively assume that $\lambda_{3}$ points along the $z$ direction (see also the discussion of Eisen and others, 2007). The directions of $\lambda_{1}$ and $\lambda_{2}$ remain undefined in the horizontal plane, but based on the vertical alignment of minima and maxima in Figure 3, we assume that they do not change with increasing depth.

Fujita and others (2006) described a method to incorporate the eigenvalues into the dielectric tensor. Power reflection coefficients (PRCs) determine the ratio of incoming and scattered fields. They can be calculated in a planar twolayer approximation for changes in the permittivity, $\varepsilon^{\prime}$, as illustrated by Paren (1981). Along the principal axes of $\lambda_{i}$ $(i=1,2,3)$, the PRC is given by

$$
r_{\lambda_{i}, z_{1}-z_{2}}=\left[\frac{\left(\lambda_{i, z_{1}}-\lambda_{i, z_{2}}\right)\left(\varepsilon_{\|}^{\prime}-\varepsilon_{\perp}^{\prime}\right)}{4 \varepsilon^{\prime}}\right]^{2} .
$$

The values of $r$ resulting from changes in eigenvalues in the $0.9 \mathrm{~m}$ intervals (upper boundary depicted with $z_{1}$, lower boundary depicted with $z_{2}$ ) are displayed in the top axis of Figure $6 \mathrm{~b}$ and $\mathrm{c}$.

For nadir incidence, the polarization of the electromagnetic wave is in the vertical plane parallel to the airplane's heading. Therefore, changes in the third eigenvector do not contribute to the scattering process in this approximation. The stronger variation of $\lambda_{1}$ in the $0.9 \mathrm{~m}$ intervals within ARZ-1 fades in ARZ-2 and simultaneously the variation of $\lambda_{2}$ increases. This directly translates into the polarization dependence of the PRCs. In ARZ-1, backscatter is largest when the polarization is parallel to the principal direction of $\lambda_{1}$; conversely, in ARZ-2 the maximum in backscatter is shifted by $90^{\circ}$ and aligns with the principal direction of $\lambda_{2}$. The differences in the corresponding PRCs reach up to $10 \mathrm{~dB}$.

\subsection{Ellipsoidal air bubbles and depth-varying COF}

We estimated the effect of ellipsoidal air bubbles with a volume-scattering model, and the effect of depth-varying COF with reflections from a stratified medium. For ARZ-1, it is not straightforward to distinguish the effect of elongated bubbles and varying cluster strength in COF in terms of their orientation, since they most likely operate in similar directions. The deformation of air bubbles in the horizontal plane, as expected from strain measurements on the surface, is indicated with the strain ellipsoid in Figure $2 \mathrm{~b}$. The direction of the long axis is almost parallel to the polarization plane with maximum backscatter in ARZ-1. The azimuth angle of the COF measurements is not constrained; however, Eisen and others (2007) inferred that the girdle at $2025 \mathrm{~m}$ depth is in the vertical plane parallel to the nearby ice divide. A recent modeling study for COF evolution at this site by Bargmann and others (2011) confirms this result, in the sense that the girdle is aligned with the principal axes of the strain ellipsoid. One of the axes is near-parallel to the ice divide. It is therefore reasonable to assume that both effects of elongated air bubbles and a varying cluster strength in COF superimpose and are aligned with the strain regime on the surface.

The estimations of both scattering coefficients and PRCs have their shortcomings, due to the applied simplifications. The volume-scattering model neglects characteristics of the finite radar pulse, as only the center frequency and scattering contributions from incoming nadir incidence are taken into account. The system's bandwidth for the short pulse is $\sim 17 \mathrm{MHz}$, and an interplay with higherfrequency components already increases the scattering coefficient by a few decibels, since the frequency enters the equations for the phase-matrix components with a power of four. The beam widening with increasing travel time may also give rise to scattering components with nonnadir incidence. This generally increases the polarization dependence. The absolute value for the scattering coefficient also heavily depends on the absorption, which dominates the extinction, since the phase-matrix components are small. Potential effects of anisotropic absorption, or a non-diagonal extinction matrix have been neglected. The bubbles were assumed to be randomly distributed. However, it is known that bubbles cluster in so-called 'cloudy bands', which leads to a non-uniform bubble distribution within the ice (Svensson and others, 2005). Ackley and Keliher (1979) took this idea further and estimated the effect of air bubbles in a way similar to our approach for the depth-varying COF. The bubble stratification was modeled with a scalar mixing formula for air and ice with a different depolarization factor for each layer due to varying bubble eccentricities. The corresponding anisotropy and backscattered power with the approach for stratified media is larger than that estimated with the volumescattering model using similar parameters. More data for air-bubble statistics in ice are needed - especially in the horizontal plane - in order to decide whether surface or volume scattering is a more suitable description. In any case, the absolute values of the scattering coefficients have a high uncertainty. This is also the case for the PRCs derived for the variations in COF: reflections from within the ice are subject to interferences, and the sample interval of the COF data is not short enough to identify potential reflection layers reliably. Changes in COF with depth in the range of the wavelength $(\sim 1 \mathrm{~m}$ in ice) may be larger than those portrayed in Figure 6. The interfaces, however, may not be planar, but could be slanted and/or rough. This substantially decreases the magnitude of the PRCs. This is to say that the absolute values of scattering coefficients and PRCs should be handled with care. Moreover, quantitative comparison of backscattered power from volume and surface scattering requires consideration of transmit and receive beam patterns, since the scattering coefficient is normalized per unit volume, while the PRCs are normalized per unit area subtended. In view of these difficulties, we do not attempt to identify the dominant backscattering mechanism on the basis of absolute backscattered power. We rather focus 
on the relative polarization dependence. The example for volume scattering in Figure $5 \mathrm{a}$ yields a $\sim 1 \mathrm{~dB}$ difference in a somewhat favorable case of fully aligned air bubbles with typical size parameters for intermediate depths. In the upper half of ARZ-1, the PRCs due to changes in eigenvalue $\lambda_{1}$ appear several decibels stronger than those caused by the changes in eigenvalue $\lambda_{2}$ (Fig. $6 b$ and c), whereas in the lower half of ARZ-1 they appear almost equal. For ARZ-2, the PRCs due to changes in $\lambda_{2}$ outweigh the PRCs due to changes in $\lambda_{1}$ by $>10 \mathrm{~dB}$. Based on the relative polarization dependence, we estimate that in ARZ-2, and probably also in ARZ-1, changes in COF are the dominant mechanisms for the observed anisotropy.

We briefly discuss the shading of ARZ-2 by ARZ-1. The increased backscatter parallel to the direction of maximal compression diminishes the transmitted power with the corresponding polarization in that direction. Therefore, polarization-dependent variations in backscattered power from ARZ-2 are to be expected, even without a specific anisotropic backscatter mechanism in that zone. To estimate this effect, we consider a lossless, stratified medium containing $n$ equidistant layers with thickness $\Delta z$ and anisotropic PRCs $r_{x}$ and $r_{y}$. The ratio, $\left|E_{\mathrm{t}, x}\right|^{2} /\left|E_{\mathrm{t}, y}\right|^{2}$, of the transmitted power after two-way penetration for the two polarization types is

$$
\frac{\left|E_{\mathrm{t}, x}\right|^{2}}{\left|E_{\mathrm{t}, y}\right|^{2}}=\left(\frac{1-r_{x}}{1-r_{y}}\right)^{2 n \Delta z},
$$

which ignores multiple reflections as they are small. To estimate an upper boundary we assume $n=900$ layers with strongly anisotropic PRCs of $r_{x}=-40 \mathrm{~dB}$ and $r_{y}=-50 \mathrm{~dB}$ with a layer thickness $\Delta z=1 \mathrm{~m}$. This extreme case results in a ratio of $-0.7 \mathrm{~dB}$, which is smaller than the ratio observed in ARZ-2.

\section{CONCLUSION}

We observe a variation in signal strength depending on the antenna orientation. The dominant symmetry has a $180^{\circ}$ periodicity, which we assign to anisotropic scattering from within the ice. To a lesser extent we also observe a $90^{\circ}$ periodicity due to birefringence caused by the macroscopic alignment of the COF. The polarization-dependent backscatter changes its direction with depth at $\sim 900 \mathrm{~m}$, coinciding with the clathrate transition and the transition from Holocene ice to ice from the last glacial (700-900 m depth). Due to a lack of data, we neglected the effect of a potentially (wind-induced) directional layer roughness and only investigated backscatter mechanisms above $900 \mathrm{~m}$ (ARZ-1) and below $900 \mathrm{~m}$ (ARZ-2) in terms of elongated air bubbles and a depth-varying COF. For ARZ-1, we propose that both effects superimpose and operate in similar directions, predetermined by the principal axes of the stress regime at the surface. We assume that bubbles are compressed parallel to the axis of maximal compression and elongated along the axis of lateral extension, causing minimum and maximum in backscatter in these directions, respectively. Similarly we hypothesize that the girdle in the COF is aligned along the same axes; this idea is supported by recent fabric-evolution modeling results in this area (Bargmann and others, 2011). The eigenvalues in ARZ-1 suggest that the horizontal width of the girdle-type COF varies with depth, increasing the backscatter perpendicular to the direction of maximal compression.
Most likely, the variations in COF dominate the observed anisotropy, since the induced polarization dependence through elongated bubbles is small. However, a potential stratification of air bubbles has been neglected and higherresolved COF measurements, as well as measurements with respect to orientation and eccentricities of the air bubbles in the horizontal plane, are needed to quantify the effects reliably. The volume scattering for anisotropic inclusions becomes more important at shallower depths when higher frequencies are used and the volume fraction of air is larger.

In ARZ-2, we propose that changes in eigenvalue $\lambda_{2}$ and a stable eigenvalue, $\lambda_{1}$, increase the backscatter in the direction normal to that observed in ARZ-1. This corresponds to the girdle to single-pole transitions suggested by Fujita and others (2003) and Matsuoka and others (2003). In contrast to ARZ-1, the variations in COF appear more clearly in the icecore data.

So far, it is unclear how the eigenvalues of COF change vertically in meter and sub-meter intervals and how the evolution is influenced by the impurity content of the ice. Given the available evidence, it is possible that the transition in anisotropic backscatter caused by changing variational patterns of COF indicates the climate transition at the end of the last glacial (the relationship with the clathrate transition at similar depths appears to be merely coincidental). To visualize the relationship of increasing dust concentration with the changing COF variations, Figure $6 \mathrm{~d}$ displays the non-sea-salt $\mathrm{Ca}^{2+}$ concentration (Fischer and others, 2007) as an impurity proxy alongside the COF measurements. For ARZ-1, the concentrations are low compared to all other depths. The exact mechanism on a grain-size scale still needs to be investigated, and it seems that the flank-flow setting is important, as Fujita and others (2006) identified anisotropic scattering near Mizuho station (converging ice-flow region), but not near Dome Fuji (located near a dome summit). More radar data are needed to determine whether the appearance of the ARZs is a universal feature in flank-flow regimes.

\section{ACKNOWLEDGEMENTS}

This work is a contribution to the European Project for Ice Coring in Antarctica (EPICA), a joint European Science Foundation/European Commission scientific program, funded by the EU and by national contributions from Belgium, Denmark, France, Germany, Italy, The Netherlands, Norway, Sweden, Switzerland and the United Kingdom. The main logistic support was provided by IPEV and PNRA (at Dome C) and AWI (at Dronning Maud Land). This is EPICA publication No. 287. Preparation of this work was supported by the Emmy Noether program of the Deutsche Forschungsgemeinschaft grant El 672/5 to O.E. and a scholarship of the Evangelisches Studienwerk e.V. Villigst to R.D. We appreciate helpful comments from Wolfgang Rack and Dale Winebrenner. Ed Waddington and an anonymous reviewer significantly improved the content and style of the manuscript.

\section{REFERENCES}

Ackley SF and Keliher TE (1979) Ice sheet internal radio-echo reflections and associated physical property changes with depth. J. Geophys. Res., 84(B10), 5675-5680

Alley RB and Fitzpatrick JJ (1999) Conditions for bubble elongation in cold ice-sheet ice. J. Glaciol., 45 (149), 147-153 
Bargmann S, Seddik H and Greve R (2011) Computational modeling of flow-induced anisotropy of polar ice for the EDML deep drilling site, Antarctica: The effect of rotation recrystallization and grain boundary migration. Int. J. Num. Anal. Meth. Geomech., 36(8) (doi: 10.1002/nag.1034)

Bendel V (2009) Untersuchung an Luftblasen im EDML-Eisbohrkern mit Bildanalyse und Ramanspektroskopie. (Master's thesis, Universität Göttingen, Fachbereich Geowissenschaften)

Birnbaum G and 14 others (2010) Strong-wind events and their influence on the formation of snow dunes: observations from Kohnen station, Dronning Maud Land, Antarctica. J. Glaciol., 56(199), 891-902 (doi: 10.3189/002214310794457272)

Dall J (2010) Ice sheet anisotropy measured with polarimetric ice sounding radar. In 30th International Geoscience and Remote Sensing Symposium (IGARSS 2010), 25-30 July 2010, Honolulu, HI, USA. Proceedings. Institute of Electrical and Electronic Engineers, Piscataway, NJ, 2507-2510

Doake CSM (1981) Polarization of radio waves in ice sheets. Geophys. J. R. Astron. Soc., 64(2), 539-558

Doake CSM, Corr HFJ and Jenkins A (2002) Polarization of radio waves transmitted through Antarctic ice shelves. Ann. Glaciol., 34, 165-170 (doi: 10.3189/172756402781817572)

Drews, R (2011) Imprints of ice dynamics and atmospheric signals on the internal structure of Antarctic ice as seen via radar. (PhD thesis, University of Bremen)

Drews R and 7 others (2009) Layer disturbances and the radio-echo free zone in ice sheets. Cryosphere, 3(2), 195-203

Eisen O, Wilhelms F, Steinhage D and Schwander J (2006) Improved method to determine radio-echo sounding reflector depths from ice-core profiles of permittivity and conductivity. J. Glaciol., 52(177), 299-310 (doi: 10.3189/172756506781828674)

Eisen O, Hamann I, Kipfstuhl S, Steinhage D and Wilhelms F (2007) Direct evidence for continuous radar reflector originating from changes in crystal-orientation fabric. Cryosphere, 1(1), 1-10

Fischer $\mathrm{H}$ and 28 others (2007) Reconstruction of millennial changes in dust emission, transport and regional sea ice coverage using the deep EPICA ice cores from the Atlantic and Indian Ocean sector of Antarctica . Earth Planet. Sci. Lett., 260(1-2), 340-354 (doi: 10.1016/j.epsl.2007.06.014)

Fujita $S$ and Mae S (1993) Relation between ice sheet internal radioecho reflections and ice fabric at Mizuho Station, Antarctica. Ann. Glaciol., 17, 269-275

Fujita S, Mae S and Matsuoka T (1993) Dielectric anisotropy in ice Ih at $9.7 \mathrm{GHz}$. Ann. Glaciol., 17, 276-280

Fujita $S$ and 6 others (1999) Nature of radio-echo layering in the Antarctic ice sheet detected by a two-frequency experiment. J. Geophys. Res., 104(B6), 13 013-13024

Fujita S, Matsuoka T, Ishida T, Matsuoka K and Mae S (2000) A summary of the complex dielectric permittivity of ice in the megahertz range and its applications for radar sounding of polar ice sheets. In Hondoh T ed. Physics of ice core records. Hokkaido University Press, Sapporo, 185-212

Fujita S, Matsuoka K, Maeno H and Furukawa T (2003) Scattering of VHF radio waves from within an ice sheet containing the verticalgirdle-type ice fabric and anisotropic reflection boundaries. Ann. Glaciol., 37, 305-316 (doi: 10.3189/172756403781815979)

Fujita S, Maeno H and Matsuoka K (2006) Radio-wave depolarization and scattering within ice sheets: a matrix-based model to link radar and ice-core measurements and its application. J. Glaciol., 52(178), 407-424 (doi: 10.3189/172756506781828548)

Hargreaves ND (1977) The polarization of radio signals in the radio echo sounding of ice sheets. J. Phys. D, 10(9), 1285-1304

Hargreaves ND (1978) The radio-frequency birefringence of polar ice. J. Glaciol., 21(85), 301-313

Hörhold W, Laepple T, Freitag J, Bigler M, Fischer H and Kipfstuhl $S$ (2012) On the impact of impurities on the densification of polar firn. Earth Planet. Sci. Lett. 325-326, 93-99 (doi: 10.1016/j.epsl.2011.12.022,M)
Karam MA and Fung AK (1983) Scattering from randomly oriented circular discs with application to vegetation. Radio Sci., 18, 557-565

Kravchenko I, Besson D, Ramos A and Remmers J (2011) Radio frequency birefringence in south polar ice and implications for neutrino reconstruction. Astropart. Phys., 34(10), 755-768

Liu C, Bentley CR and Lord NE (1994) $C$ axes from radar depolarization experiments at Upstream B Camp, Antarctica, in 1991-92. Ann. Glaciol., 20, 169-176

Matsuoka T, Fujita S, Morishima S and Mae S (1997) Precise measurement of dielectric anisotropy in ice lh at $39 \mathrm{GHz}$. J. Appl. Phys., 81(5), 2344-2348

Matsuoka K and 6 others (2003) Crystal-orientation fabrics within the Antarctic ice sheet revealed by a multi-polarization-plane and dual frequency radar survey. J. Geophys. Res., 108(B10), 2499 (doi: 10.1029/2002JB002425)

Matsuoka K, Uratsuka S, Fujita S and Nishio F (2004) Ice-flowinduced scattering zone within the Antarctic ice sheet revealed by high-frequency airborne radar. J. Glaciol., 50(170), 382-388 (doi: 10.3189/172756504781829891)

Matsuoka K, Wilen L, Hurley SP and Raymond CF (2009) Effects of birefringence within ice sheets on obliquely propagating radio waves. IEEE Trans. Geosci. Remote Sens., 475(5), 1429-1443 (doi: 10.1109/TGRS.2008.2005201)

Nakawo M, Ohmae H, Nishio F and Kameda T (1989) Dating the Mizuho 700-m core from core ice fabric data. Proc. NIPR Symp. Polar Meteorol. Glaciol., 2, 105-110

Nixdorf $U$ and 6 others (1999) The newly developed airborne radioecho sounding system of the AWI as a glaciological tool. Ann. Glaciol., 29, 231-238 (doi: 10.3189/172756499781821346)

Oerter H, Drücker C, Kipfstuhl J and Wilhelms F (2012) Kohnen station - the drilling camp for the EPICA deep ice core in Dronning Maud Land. Polarforschung, 78(1-2), 1-23

Paren JG (1981) Correspondence. Reflection coefficient at a dielectric interface. J. Glaciol., 27(95), 203-204

Reijmer CH and Van den Broeke MR (2003) Temporal and spatial variability of the surface mass balance in Dronning Maud Land, Antarctica, as derived from automatic weather stations. J. Glaciol., 49(167), 512-520 (doi: 10.3189/ 172756503781830494)

Robin GdeQ, Evans S and Bailey JT (1969) Interpretation of radio echo sounding in polar ice sheets. Philos. Trans. R. Soc. London, Ser. A, 265(1166), 437-505

Shimada W and Hondoh T (2004) In situ observation of the transformation from air bubbles to air clathrate hydrate crystals using a Mizuho ice core. J. Cryst. Growth, 265(1-2), 309-317 (doi: 10.1016/j.jcrysgro.2004.01.040)

Siegert MJ and Kwok R (2000) Ice-sheet radar layering and the development of preferred crystal orientation fabrics between Lake Vostok and Ridge B, central East Antarctica. Earth Planet. Sci. Lett., 179(2), 227-235

Steinhage D, Nixdorf U, Meyer $U$ and Miller H (1999) New maps of the ice thickness and subglacial topography in Dronning Maud Land, Antarctica, determined by means of airborne radio-echo sounding. Ann. Glaciol., 29, 267-272 (doi: 10.3189/172756499781821409)

Steinhage D, Nixdorf U, Meyer U and Miller H (2001) Subglacial topography and internal structure of central, western Dronning Maud Land, Antarctica, determined from airborne radio echo sounding. J. Appl. Geophys., 47(3-4), 183-189

Svensson A and 7 others (2005) Visual stratigraphy of the North Greenland Ice Core Project (NorthGRIP) ice core during the last glacial period. J. Geophys. Res., 110(D2), D02108 (doi: 10.1029/2004JD005134)

Thorsteinsson T (1996) Textures and fabrics in the GRIP ice core, in relation to climate history and ice deformation. Ber. Polarforsch/Rep. Pol. Res. 205

Tsang $\mathrm{L}$ and Kong JA (1978) Radiative transfer theory for active remote sensing of half-space random media. Radio Sci., 13(5), 763-773 
Tsang L, Kubasci C and Kong JA (1981) Radiative transfer theory for active remote sensing of a layer of small ellipsoidal scatterers. Radio Sci., 16(3), 321-329

Tsang L, Kong JA and Ding K-H (2000) Scattering of electromagnetic waves: theories and applications. Wiley, New York

Ueltzhöffer KJ and 6 others (2010) Distribution of air bubbles in the EDML and EDC (Antarctica) ice cores, using a new method of automatic image analysis. J. Glaciol., 56(196), 339-348 (doi: 10.3189/002214310791968511)

Waddington ED, Bolzan JF and Alley RB (2001) Potential for stratigraphic folding near ice-sheet centers. J. Glaciol., 47(159), 639-648 (doi: 10.3189/172756501781831756)

Wang B, Tian G, Cui X and Zhang X (2008) The internal COF features in Dome $\mathrm{A}$ of Antarctica revealed by multi-polarization-plane RES. Appl. Geophys., 5(3), 230-237

Wesche C, Eisen O, Oerter H, Schulte D and Steinhage D (2007) Surface topography and ice flow in the vicinity of the EDML deep-drilling site, Antarctica. J. Glaciol., 53(182), 442-448 (doi: 10.3189/002214307783258512)

Wilen LA, DiPrinzio CL, Alley RB and Azuma N (2003) Development, principles, and applications of automated ice fabric analyzers. Microsc. Res. Techn., 62(1), 2-18

Wilhelms F (2000) Messung dielektrischer Eigenschaften polarer Eiskerne. Ber. Polarforsch/Rep. Pol. Res. 367

Wilhelms F, Kipfstuhl J, Miller H, Heinloth K and Firestone J (1998) Precise dielectric profiling of ice cores: a new device with improved guarding and its theory. J. Glaciol., 44(146), 171-174

Wilson CJL, Russell-Head DS and Sim HM (2003) The application of an automated fabric analyzer system to the textural evolution of folded ice layers in shear zones. Ann. Glaciol., 37, 7-17 (doi: 10.3189/172756403781815401)

\section{APPENDIX}

The calculation of the phase and extinction matrix for ellipsoidal scatterers has been demonstrated by Tsang and others (1981) and modified by Karam and Fung (1983). We adapt the geometry so it contains two half-spaces (labeled 0 and 1) representing air and ice, respectively. The change of geometry involves changing boundary conditions which alter the final results slightly. The boundary conditions used here were also used by Tsang and Kong (1978). We outline the calculations.

Equation (1) can be split into two equations (upward/downward radiation):

$$
\begin{gathered}
\frac{\mathrm{d} \mathbf{l}(\theta, \phi, \mathbf{z})}{\sec (\theta) \mathrm{d} z}=-\underline{\mathbf{k}}_{\mathbf{e}}(\theta, \phi) \mathbf{I}(\theta, \phi, z)+\mathbf{S}_{\mathbf{u}} \\
\frac{\mathrm{d} \mathbf{I}(\pi-\theta, \phi, \mathbf{z})}{\sec (\theta) \mathrm{d} z}=\underline{\mathbf{k}}_{\mathbf{e}}(\pi-\theta, \phi) \mathbf{I}(\pi-\theta, \phi, z)-\mathbf{S}_{\mathbf{d}}
\end{gathered}
$$

where $\pi-\theta$ stands for the downward direction. In this approximation the extinction matrix is assumed to be diagonal. The source terms $\mathbf{S}_{\mathbf{u}}$ and $\mathbf{S}_{\mathbf{d}}$ mark the amount of radiation scattered in the upward $(\mathrm{u})$ and downward (d) direction, respectively. They are mainly determined by the phase matrix

$$
\begin{aligned}
\mathbf{S}_{\mathbf{u}}= & \int \mathrm{d} \Omega^{\prime} \underline{\mathbf{P}}\left(\theta, \phi, \theta^{\prime}, \phi^{\prime}\right) \mathbf{I}\left(\theta^{\prime}, \phi^{\prime}\right) \\
& +\underline{\mathbf{P}}\left(\theta, \phi, \pi-\theta^{\prime}, \phi^{\prime}\right) \mathbf{I}\left(\pi-\theta^{\prime}, \phi^{\prime}\right), \\
\mathbf{S}_{\mathbf{d}}= & \int \mathrm{d} \Omega^{\prime} \underline{\mathbf{P}}\left(\pi-\theta, \phi, \theta^{\prime}, \phi^{\prime}\right) \mathbf{I}\left(\theta^{\prime}, \phi^{\prime}\right) \\
& +\underline{\mathbf{P}}\left(\pi-\theta, \phi, \pi-\theta^{\prime}, \phi^{\prime}\right) \mathbf{I}\left(\pi-\theta^{\prime}, \phi^{\prime}\right) .
\end{aligned}
$$

The phase matrix components are given by Tsang and others (1981) and calculated using scattering dyads which include the half-axes describing the ellipsoid. They also contain a prefactor with wavelength of the radar system and volume fraction of the air bubbles in ice. The dashed angles mark the incoming, the non-dashed angles the scattered directions (Fig. 4). The degree of alignment of the inclusions is considered by averaging the matrix elements over an orientation density distribution, $\psi(\alpha, \beta, \gamma)$, for the Euler angles $\alpha, \beta$ and $\gamma$. This distribution is not yet known from ice-core data. To simplify the (numerical) integration we use a box-like shape of

$\psi(\alpha, \beta, \gamma, \Delta \alpha, \Delta \beta, \Delta \gamma)=\left\{\begin{array}{cl} & \alpha \in[\alpha-\Delta \alpha, \alpha+\Delta \alpha] \\ \frac{1}{8 \Delta \alpha \Delta \beta \Delta \gamma} & \beta \in[\beta-\Delta \beta, \beta+\Delta \beta] \\ & \gamma \in[\gamma-\Delta \gamma, \gamma+\Delta \gamma] \\ 0 & \text { otherwise }\end{array}\right.$

which results in a mere change of integration boundaries during the integration:

$$
P_{m n}(\theta, \phi)=\int \mathrm{d} \alpha \mathrm{d} \beta \mathrm{d} \gamma \psi(\alpha, \beta, \gamma) P_{m n}(\alpha, \beta, \gamma, \theta, \phi)
$$

with $m, n=1 \ldots 4$ depicting the different matrix components ( $m$ is also used below to mark components of vectors). At this step, averaging over a size or eccentricity distribution is also possible. The first two diagonal elements of the extinction matrix are given by

$$
\begin{aligned}
k_{\mathrm{v}} & =k_{a b}+k_{\mathrm{sv}}\left(\Omega^{\prime}\right) \\
& =2(1-\mathrm{vol}) \operatorname{Im}\left(k_{1}\right)+\int \mathrm{d} \Omega P_{11}\left(\Omega, \Omega^{\prime}\right)+P_{21}\left(\Omega, \Omega^{\prime}\right) \\
k_{\mathrm{h}} & =k_{a b}+k_{\mathrm{sh}}\left(\Omega^{\prime}\right) \\
& =2(1-\mathrm{vol}) \operatorname{Im}\left(k_{1}\right)+\int \mathrm{d} \Omega P_{12}\left(\Omega, \Omega^{\prime}\right)+P_{22}\left(\Omega, \Omega^{\prime}\right),
\end{aligned}
$$

which assumes there is no anisotropy in absorption. The inclusion of absorption influences the magnitude of the scattering coefficient and introduces the dependency on the volume fraction (vol) which cancels otherwise (see also Karam and Fung, 1983). The system of Eqns (A1) and (A2) with transmissivity $\left(\underline{\mathbf{T}}_{\mathbf{0 1}}\right)$ and reflectivity $\left(\underline{\mathbf{R}}_{\mathbf{1 1}}\right)$ matrices and the boundary condition

$$
\mathbf{I}_{\mathbf{d}}(z=0)=\underline{\mathbf{T}}_{\mathbf{0 1}} \mathbf{I}_{\mathbf{0}}(z=0)+\underline{\mathbf{R}}_{\mathbf{1 1}} \mathbf{I}_{\mathbf{u}}(z=0)
$$

can be turned into a system of integral equations (using an integrating factor):

$$
\begin{aligned}
I_{\mathrm{u}, m}(z) & =\int_{-\infty}^{z} \mathrm{~d} z^{\prime} \mathrm{e}^{-\frac{k_{\mathrm{e}, m m}}{\cos (\theta)}\left(z-z^{\prime}\right)} \frac{S_{\mathrm{u}, m}(\theta, \phi)}{\cos (\theta)} \\
I_{\mathrm{d}, m}(z) & =\int_{z}^{0} \mathrm{~d} z^{\prime} \frac{S_{\mathrm{d}, m}}{\cos (\theta)} \mathrm{e}^{\frac{k_{\mathrm{e}, m m}(\cos (\theta)}{\left(z-z^{\prime}\right)}} \\
& +\underline{\mathbf{T}}_{\mathbf{0 1}} \mathbf{I}_{\mathbf{0}}(z=0)_{m} \mathrm{e}^{\frac{k_{\mathrm{e}, m m}}{\cos (\theta)} z} \\
& +\left[\underline{\mathbf{R}}_{\mathbf{1 1}} \int_{-\infty}^{0} \mathrm{~d} z^{\prime} \mathrm{e}^{\frac{k_{\mathrm{e}, m m}}{\cos (\theta)}\left(z-z^{\prime}\right)} \frac{\mathbf{S}_{\mathrm{u}}(\theta, \phi)}{\cos (\theta)}\right]_{m} .
\end{aligned}
$$

The incoming intensity originates from a single direction only:

$$
\mathbf{I}_{0}(\pi-\theta, \phi)=\mathbf{I}_{\mathbf{i n c}} \delta\left(\cos \left(\theta_{0}\right)-\cos \left(\theta_{0 \mathrm{i}}\right)\right) \delta\left(\phi_{0}-\phi_{0 \mathrm{i}}\right)
$$


with $\delta$ representing the delta function. Once the upward intensity at $z=0$ is determined, the outgoing intensity is

$$
\mathbf{I}_{\mathbf{0}}\left(\theta_{\mathrm{OS}}, \phi_{\mathrm{OS}}\right)=\underline{\mathbf{T}}_{\mathbf{1 0}} \mathbf{I}\left(\theta_{\mathrm{s}}, \phi_{\mathrm{s}}, z=0\right) \text {. }
$$

Equations (A8) and (A9) can be solved iteratively by assuming the scattering part, $\mathbf{S}_{\mathbf{d}}, \mathbf{S}_{\mathbf{u}}$, to be small. We mark the individual terms of the perturbation series with superscripts according to the order. In zeroth order, scattering is entirely neglected, therefore

$$
\begin{aligned}
& l_{\mathrm{u}, m}^{(0)}(z)=0 \\
& l_{\mathrm{d}, m}^{(0)}(z)=\left(\underline{\mathbf{T}}_{\mathbf{0 1}} \mathbf{I}_{\mathbf{0}}(z=0)\right)_{m} \mathrm{e}^{\frac{k_{\mathrm{e}, m m}}{\cos (\theta)} z} .
\end{aligned}
$$

This means the incoming wave dominates, there is no volume scattering and the downwards radiation is simply attenuated. For the first-order approximation we use the zeroth-order solution in the scattering term of Eqns (A8) and (A9):

$$
\begin{aligned}
& \frac{l_{\mathrm{u}, m}^{(1)}(z)}{\sec (\theta)}= \\
& \int_{-\infty}^{z} \mathrm{~d} z^{\prime} \mathrm{e}^{-\frac{k_{e}, m m}{\cos (\theta)}\left(z-z^{\prime}\right)} \int \mathrm{d} \Omega^{\prime}\left[\underline{\mathbf{P}}\left(\theta, \phi, \pi-\theta^{\prime}, \phi^{\prime}\right) \underline{\mathbf{I}}_{\mathbf{0 1}} \mathbf{I}_{\mathbf{0}}\right]_{m} \mathrm{e}^{\frac{k_{e}, m m}{\cos (\theta)} z^{\prime}} \\
& =\int \mathrm{d} \Omega^{\prime}\left[\underline{\mathbf{P}}\left(\theta, \phi, \pi-\theta^{\prime}, \phi^{\prime}\right) \underline{\mathbf{I}}_{\mathbf{0 1}} \mathbf{I}_{\mathbf{0}}\right]_{m} \frac{\mathrm{e}^{k_{\mathrm{e}, m m} \sec \left(\theta^{\prime}\right) z}}{k_{\mathrm{e}, m m}\left(\sec (\theta)+\sec \left(\theta^{\prime}\right)\right)}
\end{aligned}
$$

$$
I_{u, m}^{(1)}
$$$$
\frac{I_{\mathrm{u}, m, z=0}^{(1)}}{\sec (\theta)}=
$$$$
\int \mathrm{d} \Omega^{\prime}\left[\underline{\mathbf{P}}\left(\theta, \phi, \pi-\theta^{\prime}, \phi^{\prime}\right) \underline{\mathbf{I}}_{\mathbf{0 1}} \mathbf{I}_{\mathbf{i n c}}\right] m \frac{\delta\left[\cos \left(\theta_{\mathrm{O}}\right)-\cos \left(\theta_{\mathrm{oi}}\right)\right] \delta\left(\phi_{\mathrm{O}}-\phi_{\mathrm{oi}}\right)}{k_{\mathrm{e}, m m}\left[\sec (\theta)+\sec \left(\theta^{\prime}\right)\right]} .
$$

In order to make use of the delta functions we substitute $\mathrm{d} \theta^{\prime}$ with $\operatorname{dcos}\left(\theta^{\prime}\right)$ and relate the incoming angles, $\theta_{0}$ and $\phi_{\mathrm{O}}$, in medium 0 via Snell's law to the angles in medium 1, $\mathrm{d} \theta^{\prime} \sin \left(\theta^{\prime}\right)=\mathrm{d} \theta_{\mathrm{O}} 0^{\prime} \sin \left(\theta_{\mathrm{O}}^{\prime}\right) \frac{\varepsilon_{0} \cos \left(\theta_{0}^{\prime}\right)}{\varepsilon_{1} \cos \left(\theta^{\prime}\right)}:$

$I_{\mathrm{u}, m, z=0}^{(1)}=\sec (\theta) \frac{\varepsilon_{0}}{\varepsilon_{1}} \int_{0}^{2 \pi} \mathrm{d} \phi^{\prime} \int_{1}^{0} \mathrm{~d} \cos \left(\theta_{\mathrm{O}}^{\prime}\right) \sec \left(\theta^{\prime}\right) \cos \left(\theta_{\mathrm{O}}^{\prime}\right)$

$\left[\underline{\mathbf{P}}\left(\theta, \phi, \pi-\theta^{\prime}, \phi^{\prime}\right) \underline{\mathbf{I}}_{\mathbf{0 1}} \mathbf{I}_{\mathbf{i n c}}\right]_{m} \frac{\delta\left(\cos \left(\theta^{\prime}\right)-\cos \left(\theta_{\mathrm{i}}^{\prime}\right)\right) \delta\left(\phi^{\prime}-\phi_{\mathrm{i}}^{\prime}\right)}{k_{\mathrm{e}, m m}\left(\sec (\theta)+\sec \left(\theta^{\prime}\right)\right)}$

$=\frac{\varepsilon_{0}}{\varepsilon_{1}}\left[\underline{\mathbf{P}}\left(\theta, \phi, \pi-\theta^{\prime}, \phi^{\prime}\right) \underline{\mathbf{I}}_{\mathbf{0 1}} \mathbf{l}_{\mathbf{i n c}}\right]_{m} \frac{\sec (\theta) \cos \left(\theta_{\mathrm{Oi}}\right)}{k_{\mathrm{e}, m m}\left(\sec (\theta)+\sec \left(\theta_{\mathrm{i}}\right) \cos \left(\theta_{\mathrm{i}}\right)\right)}$.

(A16)

Using the boundary condition, Eqn (A11), the first-order approximation is

$$
\begin{aligned}
& I\left(\theta_{\mathrm{s}}, \phi_{\mathrm{s}}\right)_{\mathrm{u}, m, z=0}^{(1)}=\frac{\varepsilon_{0}}{\varepsilon_{1}} \frac{\sec \left(\theta_{\mathrm{s}}\right) \cos \left(\theta_{\mathrm{oi}}\right)}{k_{\mathrm{e}, m m}\left(\sec \left(\theta_{\mathrm{s}}\right)+\sec \left(\theta_{\mathrm{i}}\right)\right) \cos \left(\theta_{\mathrm{i}}\right)} \\
& {\left[\underline{\mathbf{T}}_{\mathbf{1 0}} \underline{\mathbf{P}}\left(\theta_{\mathrm{s}}, \phi_{\mathrm{s}}, \pi-\theta_{\mathrm{i}}, \phi_{\mathrm{i}}\right) \underline{\mathbf{I}}_{\mathbf{0 1}} \mathbf{I}_{\mathbf{i n c}}\right]_{m} .}
\end{aligned}
$$

The bistatic scattering coefficient becomes the backscattering coefficient for $\theta_{\mathrm{os}}=\theta_{\mathrm{oi}}, \phi_{\mathrm{os}}=\pi+\phi_{\mathrm{oi}}$. Therefore by using Eqn (2):

$\sigma_{\mathrm{vv}}^{(1)}=2 \pi t_{\mathrm{v} 10}^{2}\left(\theta_{\mathrm{i}}\right) \frac{\cos \left(\theta_{\mathrm{oi}}\right)^{2} \varepsilon_{0}}{\cos \left(\theta_{\mathrm{i}}\right) \varepsilon_{1}} \frac{P_{11}\left(\theta_{\mathrm{i}}, \pi+\phi_{\mathrm{i}}, \pi-\theta_{\mathrm{i}}, \phi_{\mathrm{i}}\right)}{k_{\mathrm{v}}}$,

$\sigma_{\mathrm{hh}}^{(1)}=2 \pi t_{\mathrm{h} 10}^{2}\left(\theta_{\mathrm{i}}\right) \frac{\cos \left(\theta_{\mathrm{oi}}\right)^{2} \varepsilon_{0}}{\cos \left(\theta_{\mathrm{i}}\right) \varepsilon_{1}} \frac{P_{22}\left(\theta_{\mathrm{i}}, \pi+\phi_{\mathrm{i}}, \pi-\theta_{\mathrm{i}}, \phi_{\mathrm{i}}\right)}{k_{\mathrm{h}}}$,

$\sigma_{\mathrm{hv}}^{(1)}=2 \pi t_{\mathrm{v} 10}\left(\theta_{\mathrm{i}}\right) t_{\mathrm{h} 01}\left(\theta_{\mathrm{i}}\right) \frac{\cos \left(\theta_{\mathrm{oi}}\right)^{2} \varepsilon_{0}}{\cos \left(\theta_{\mathrm{i}}\right) \varepsilon_{1}} \frac{P_{12}\left(\theta_{\mathrm{i}}, \pi+\phi_{\mathrm{i}}, \pi-\theta_{\mathrm{i}}, \phi_{\mathrm{i}}\right)}{k_{\mathrm{h}}}$,

$\sigma_{\mathrm{vh}}^{(1)}=2 \pi t_{\mathrm{h} 10}\left(\theta_{\mathrm{i}}\right) t_{\mathrm{v} 01}\left(\theta_{\mathrm{i}}\right) \frac{\cos \left(\theta_{\mathrm{oi}}\right)^{2} \varepsilon_{0}}{\cos \left(\theta_{\mathrm{i}}\right) \varepsilon_{1}} \frac{P_{21}\left(\theta_{\mathrm{i}}, \pi+\phi_{\mathrm{i}}, \pi-\theta_{\mathrm{i}}, \phi_{\mathrm{i}}\right)}{k_{\mathrm{v}}}$.

Integrating the phase matrix elements over the Euler angles and the solid angle, $d \Omega$, results in a fivefold integral. Analytically, this case is only feasible for the case of fully aligned spheroids. This serves as a cross check for the numerical solution. The numerical integration is implemented with a Monte Carlo integration scheme. 